\title{
Modelling the transmission and control strategies of varicella in Shenzhen
}

\author{
Xiujuan Tang* \\ Shenzhen center for disease control and prevention, Shenzhen, China
}

\section{Objective}

To model the transmission dynamics of varicella among school children in Shenzhen, to determine the effect of the school-based vaccination intervention.

\section{Introduction}

Varicella (chickenpox) is a highly transmissible childhood disease. Between 2010 and 2015, it displayed two epidemic waves annually among school populations in Shenzhen, China. However, their transmission dynamics remain unclear and there is no school-based vaccination program in Shenzhen to-date. In this study, we developed a mathematical model to compare a school-based vaccination intervention scenario with a baseline (i.e. no intervention)scenario.

\section{Methods}

Data on varicella reported cases were downloaded from the Infectious Disease Reporting Information Management System. We obtained the population size, age structure of children aged 15 or under, the class and school distribution from Shenzhen Education Bureau. We developed an Agent-Based Susceptible-ExposedInfectious-Recovered (ABM-SEIR) Model that considered withinclass, class-to-class and out-of-school transmission modes. The intervention scenario was that school-wide vaccination intervention occurred when an outbreak threshold was reached within a school. We varied this threshold level from five to ten cases. We compared the reduction of disease outbreak size and estimated the key epidemiological parameters under the intervention strategy.

\section{Results}

Our ABM-SEIR model provided a good model fit to the two annual varicella epidemic waves from 2013 to 2015. The transmission dynamics displayed strong seasonality. Our results suggested that a school-based vaccination strategy could effectively prevent large outbreaks at different thresholds.

\section{Conclusions}

There was a considerable increase in reported varicella cases from 2013 to 2015 in Shenzhen. Our modelling study provided important theoretical support for disease control decision making during school outbreaks and the development of a school-based vaccination programme.

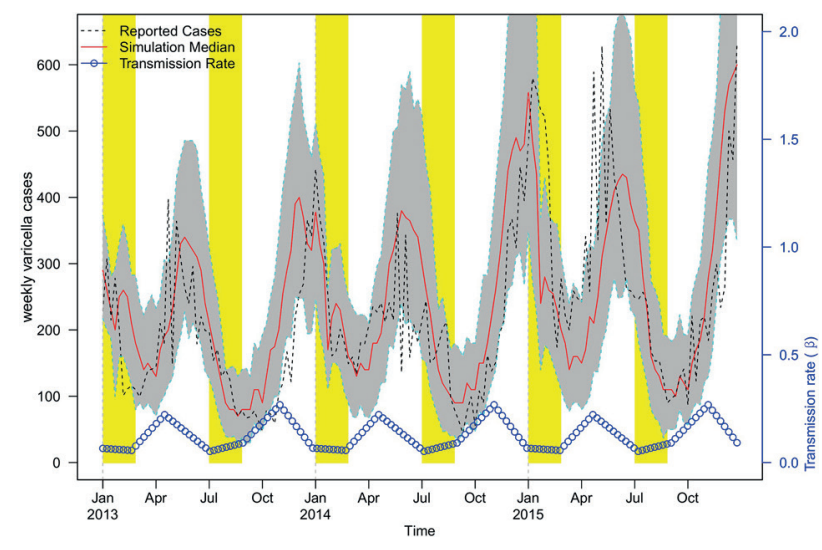

Fig 1. The ABM simulation results of varicella reported cases in Shenzhen from 2013 to 2015. The simulation median is plotted in red, reported cases are in black dashed line, the fitted transmission rate, $\beta(\mathrm{t})$, is the blue line at the bottom and the $95 \%$ Confidence Interval (C.I.) is in grey. School holidays are shaded in yellow.
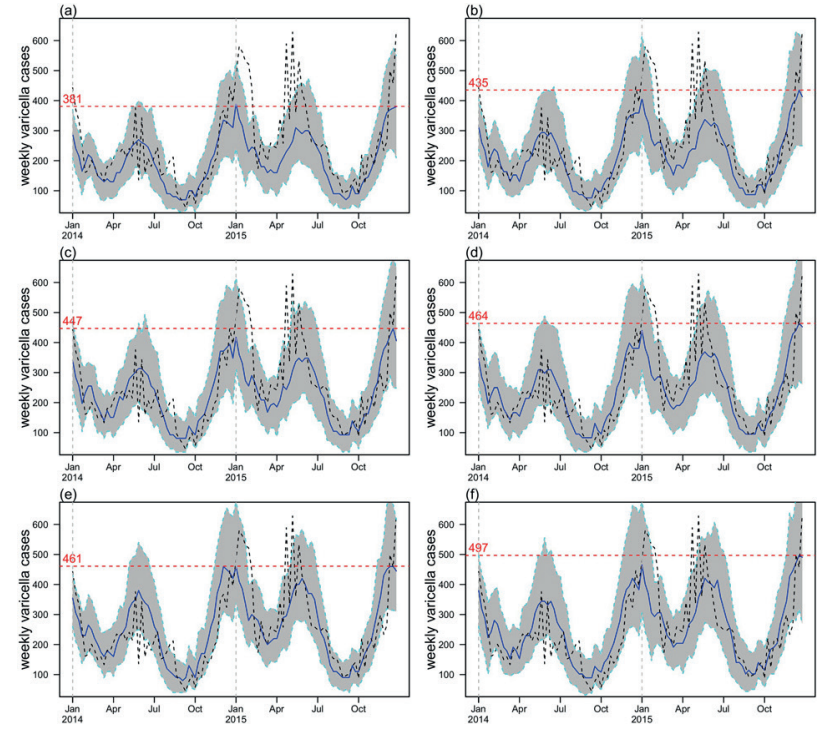

Fig 2. Simulation results with vaccination strategy from 2014-2015. The black dashed line is the confirmed cases which could be regarded as the baseline (i.e. no intervention) scenario. Simulation median is plotted in blue with $90 \%$ C.I. in grey. Panel (a), (b), (c), (d), (e) and (f) are simulation results with vaccination threshold set to be 5, 6, 7, 8, 9 and 10 (cases per week per school) respectively. The red dashed lines are the maximum weekly varicella cases during the simulation period (blue line), which represents the outbreak size under different outbreak thresholds.

\section{Keywords}

varicella; transmission; Modelling 